\title{
Manifestações bucais em pacientes pediátricos infectados pelo HIV: uma revisão sistemática da literatura
}

\author{
Oral manifestations in pediatric HIV infected patients: \\ a systematic literature review
}

\author{
Stéphanie Quadros Tonelli* \\ Wallace de Freitas Oliveira** \\ Gabriela Araújo Oliveira** \\ Daniela Araújo Veloso Popoff ${ }^{* * * *}$ \\ Mânia de Quadros Coelho ${ }^{* * * * *}$ \\ Edwaldo de Souza Barbosa Júnior ${ }^{* * * *}$
}

\section{Resumo}

Introdução: a Síndrome da Imunodeficiência Adquirida (Sida/Aids), quando ocorre em indivíduos de $\mathrm{O}$ a 13 anos de idade, é denominada de Aids pediátrica e pode apresentar manifestações significativas na cavidade bucal. Objetivo: este estudo teve o propósito de investigar, mediante uma revisão sistemática da literatura, as principais manifestações bucais nos pacientes pediátricos infectados pelo HIV. Métodos: por meio de busca nas bases DOAJ (Directory of Open Access Journals), SciELO e BVS (Biblioteca Virtual em Saúde - Bireme), foram selecionados onze artigos publicados entre 2008 e 2013. Resultados: na avaliação realizada através da escala de Jadad, seis dos nove estudos analíticos obtiveram pontuação igual ou maior que três, sendo considerados de boa expressividade metodológica. As principais manifestações bucais presentes em pacientes pediátricos infectados pelo HIV encontradas nos artigos selecionados foram: cárie, candidose, queilite angular, eritema linear gengival, lesões na mucosa, patologias nas glândulas salivares, sarcoma de Kaposi, linfoma não Hodgkin, doenças fúngicas, doenças virais, doença periodontal, hipoplasia de esmalte e condiloma acuminado. Conclusão: evidenciou-se, por meio deste estudo, a importância do cirurgião-dentista em equipes multiprofissionais no atendimento a essas crianças, bem como a realização de mais estudos que reportem sobre as condições bucais de crianças infectadas pelo HIV.

Palavras-chave: HIV. Odontopediatria. Pediatria. Manifestações bucais.

\section{Introdução}

A Síndrome da Imunodeficiência Adquirida (Sida/Aids) é uma condição sistêmica de deficiência imunológica causada pelo Vírus da Imunodeficiência Humana (HIV), que pertence à família Retroviridae, podendo ser transmitido pela via sanguínea, pelo contato sexual e pela via vertical - durante a gestação, o parto ou a amamentação ${ }^{1}$. A Aids é considerada pediátrica quando a infecção pelo HIV ocorre de 0 a 13 anos de idade; acima de 13 anos os pacientes são incluídos nas estatísticas de adultos, por apresentarem padrões semelhantes ${ }^{1-3}$.

Graduanda do curso de Odontologia da Universidade Estadual de Montes Claros (Unimontes), Montes Claros, MG.

Graduando do curso de Odontologia da Unimontes, Montes Claros, MG.

Graduanda do curso de Odontologia da Unimontes, Montes Claros, MG.

**** Professora (com pós-doutorado) do curso de Graduação em Odontologia da Unimontes, Montes Claros, MG.

***** Professora mestra da Clínica Integrada IV - Atendimento a pacientes HIV-soropositivos do curso de Graduação em Odontologia da Unimontes, Montes Claros, MG.

****** Professor mestre da Clínica Integrada IV - Atendimento a pacientes HIV-soropositivos do curso de Graduação em Odontologia da Unimontes, Montes Claros, MG. 
Os sintomas da doença são distintos entre adultos e crianças, tendo em vista que os pacientes infantis possuem o sistema imunológico imaturo, o que leva a uma maior deficiência da defesa frente às infecções ${ }^{1}$. O primeiro relato de transmissão perinatal registrada no Brasil data de $1985^{2}$. Esse tipo de transmissão representa $85 \%$ dos casos pediátricos notificados nos Estados Unidos e ao redor do mundo, os $15 \%$ restantes incluem crianças com hemofilia, desordens de coagulação, receptores de transfusões de sangue e outros riscos não identificados ${ }^{1,3}$.

A infecção pelo HIV/AIDS em crianças e adolescentes é considerada um problema de saúde pública mundial há mais de uma década, e, cada vez mais, são necessários estudos mais aprofundados no intuito de se promover uma melhor qualidade de vida para esses pacientes ${ }^{1}$.

A infecção pelo HIV é caracterizada pelo aparecimento de um conjunto de doenças consideradas como seus indicadores, podendo ser em forma de manifestações bucais, tais como candidíase oral, herpes simples, queilite angular, leucoplasia pilosa, doença periodontal, dentre outras ${ }^{1,3-7}$. Essas manifestações são muito comuns e, na maior parte das vezes, são os primeiros sinais e sintomas da doença, e o cirurgião-dentista é, nesses casos, o primeiro a se deparar com o fato ${ }^{3}$.

Em razão da importância do tema, este estudo teve o propósito de investigar, mediante uma revisão sistemática da literatura, as principais manifestações bucais nos pacientes pediátricos infectados pelo HIV, cujos resultados auxiliarão na atualização profissional e, sobretudo, contribuirão para a promoção da saúde bucal e melhoria da qualidade de vida dessas crianças.

\section{Métodos}

Este estudo consistiu em uma revisão sistemática da literatura sobre as principais manifestações bucais nos pacientes pediátricos infectados pelo HIV.

\section{Planejamento do estudo e estratégia de pesquisa}

Trata-se de estudo com caráter descritivo, que compreendeu o levantamento de referencial realizado nas bases de dados eletrônicas, nacionais e internacionais, DOAJ (Directory of Open Access Journals), SciELO e BVS (Biblioteca Virtual em Saúde Bireme), por meio da consulta pelos descritores $H I V$ AND Pediatric AND pediatric dentistry.

$\mathrm{O}$ acesso aos artigos completos foi realizado através do portal da Capes (www.periodicos.capes.gov. br). Os artigos identificados pela estratégia de busca foram avaliados, de forma independente e cega, por dois pesquisadores (autores), obedecendo rigorosamente aos critérios de inclusão. A seleção dos artigos foi feita a partir dos títulos e resumos, e a busca foi realizada durante os meses de abril e maio de 2013.

\section{Critérios de inclusão dos estudos selecionados}

Foram selecionados trabalhos que estivessem dentro dos seguintes critérios: textos disponíveis na íntegra; período de publicação (janeiro de 2008 a março de 2013); relação direta com a Odontologia; população-alvo (crianças); idioma (português, inglês e espanhol). Não houve restrições sobre o tipo de estudo e amostra. Foram excluídos os estudos que não obedeceram aos critérios de inclusão supracitados e estudos encontrados em mais de uma base de dados.

\section{Avaliação da qualidade metodológica}

Para avaliar a qualidade das pesquisas analíticas incluídas no estudo, foi usada a Escala de Qualidade de $\mathrm{Jadad}^{8}$, que consiste em cinco critérios com pontuação total de 0 a 5 pontos. Pesquisas com pontuações inferiores a 3 pontos são consideradas de baixa qualidade metodológica e com poucas possibilidades de extrapolação dos resultados para a prática clínica. $\mathrm{O}$ Quadro 1 mostra a pontuação dos estudos analíticos selecionados de acordo com esse instrumento. 


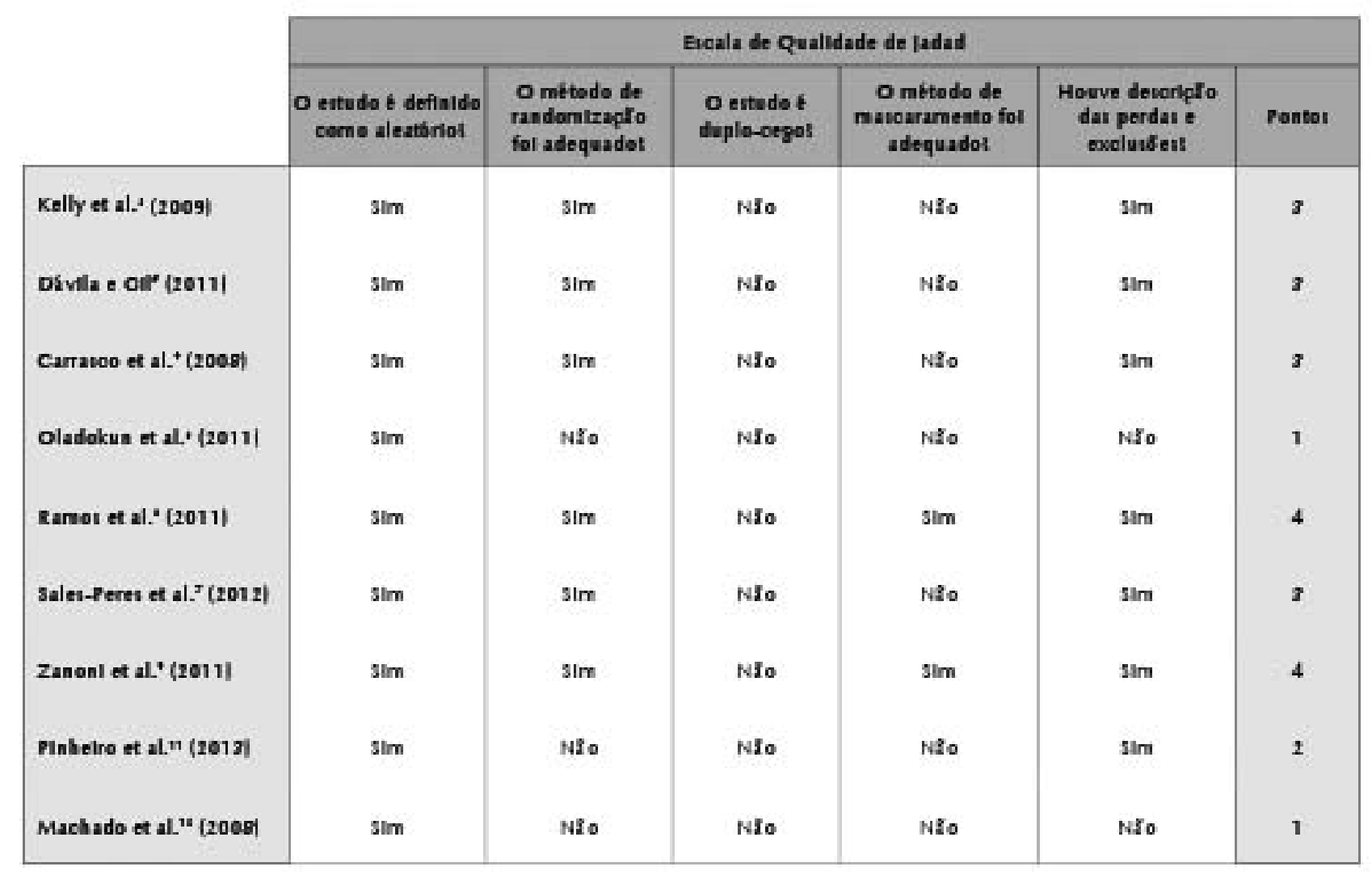

\section{Resultados}

Na combinação dos descritores HIV AND Pediatric AND pediatric dentistry, foi encontrado um total de 95 artigos, sendo 36 na DOAJ; 35 na base SciELO e 24 na BVS - Bireme. Após a supressão das duplicatas, dos artigos que não se encaixavam nos critérios de inclusão e cuja temática não contemplava a abordagem deste estudo, permaneceram onze artigos.

A realização de metanálise não foi possível devido à heterogeneidade dos estudos, impossibilitando uma comparação. Assim, foi feita a descrição individual, exposta no Quadro 2, com a apresentação das principais características dos estudos selecionados. Os estudos selecionados foram realizados em diferentes países, sendo cinco no Brasil, dois na Venezuela e um estudo em cada um destes países: África do Sul, USA, Moçambique e Nigéria. Com relação ao ano de publicação, os estudos se encontraram na faixa de 2008 a 2013.
$\mathrm{Na}$ avaliação realizada através da escala de $\mathrm{Jadad}^{8}$, seis dos nove estudos analíticos obtiveram pontuação igual ou maior que três, sendo considerados de boa expressividade metodológica.

De uma forma geral, as principais manifestações bucais presentes em pacientes pediátricos infectados pelo HIV encontradas nos artigos selecionados foram: cárie, candidose, queilite angular, eritema linear gengival, lesões na mucosa, patologias nas glândulas salivares, Sarcoma de Kaposi, Linfoma não Hodgkin, doenças fúngicas, doenças virais, doença periodontal, hipoplasia de esmalte e condiloma acuminado , $3-7,7-11 .^{\text {. }}$

Com relação às inferências deixadas pelos artigos, pode-se dizer que os pacientes pediátricos infectados pelo HIV, comumente, apresentam algum tipo de manifestação bucal em fases não específicas de sua infância, apesar de terem sido encontrados poucos estudos que correlacionam a temática com a Odontologia ${ }^{1-7,9-12}$. 


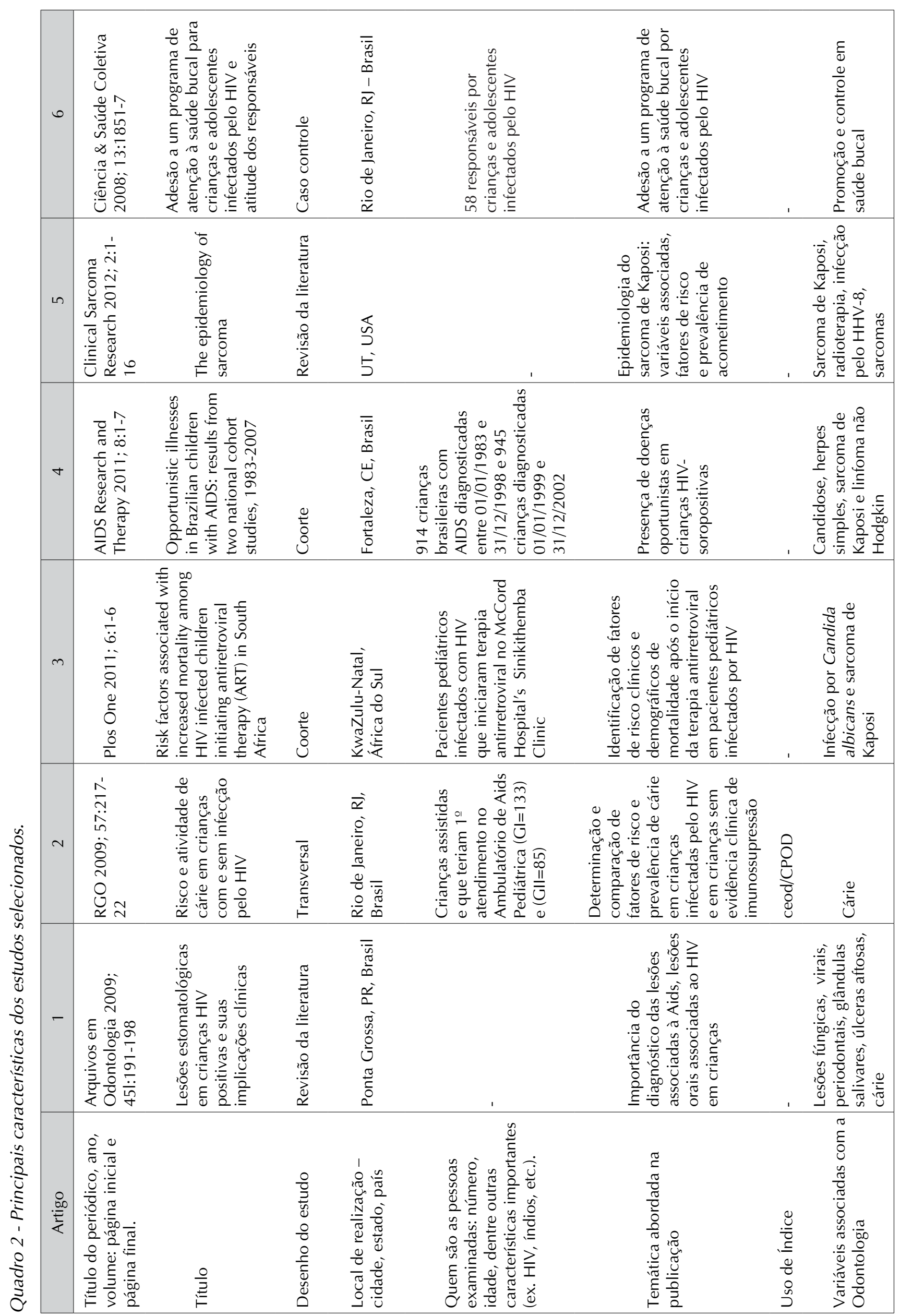




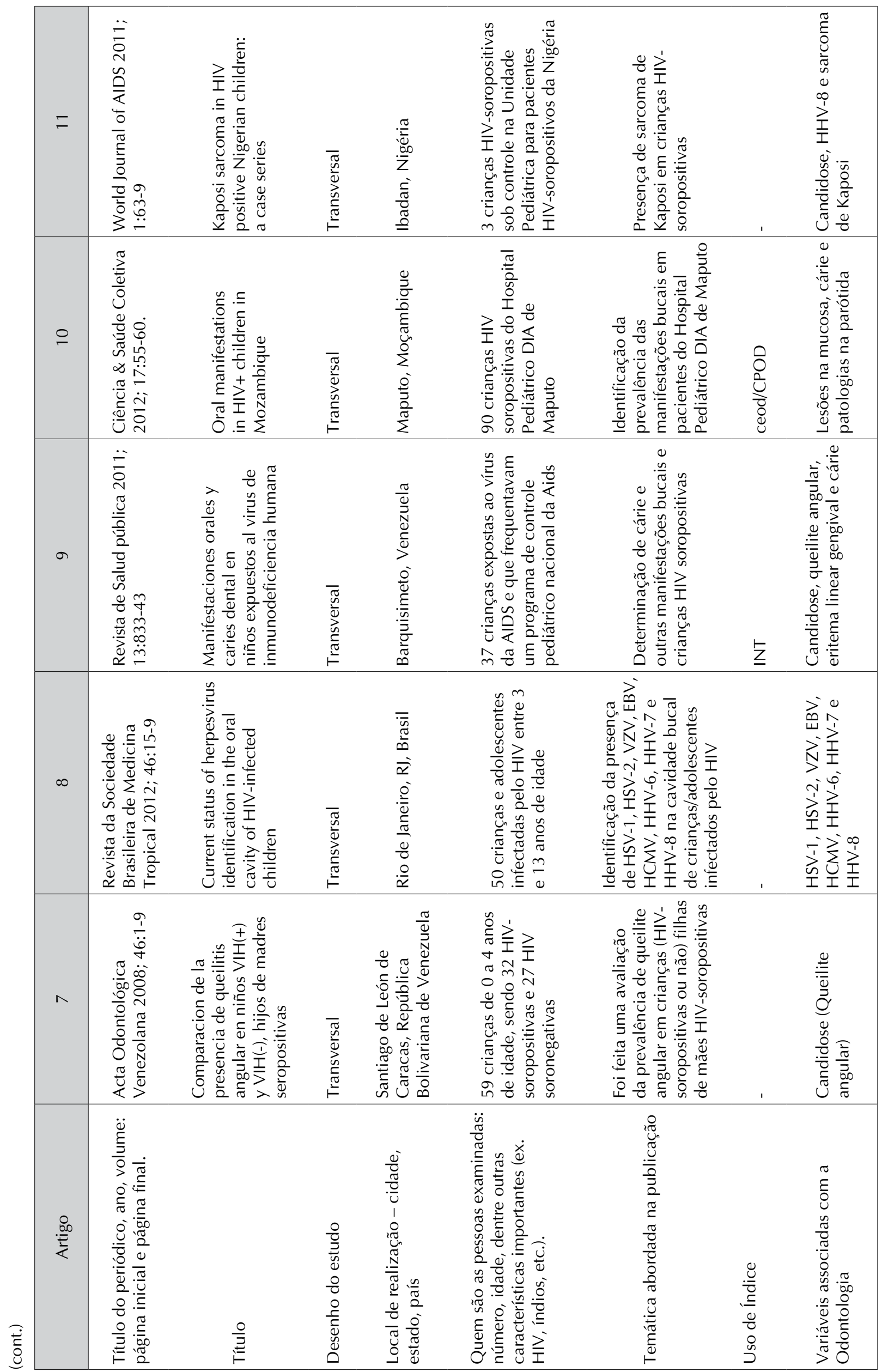




\section{Discussão}

De acordo com os resultados, cerca de 85 a 91,8\% das crianças adquirem o vírus a partir de suas mães, por meio da transmissão vertical ${ }^{1-3}$.

Lesões bucais são comuns nos pacientes pediátricos infectados pelo $\mathrm{HIV}^{1,11}$. O paciente pediátrico pode apresentar lesões na mucosa bucal como manifestações secundárias, as quais sinalizam a progressão da doença, a ineficácia do tratamento antirretroviral, assim como essas lesões podem preceder as manifestações sistêmicas da Aids ${ }^{1,3,6,7,11}$.

A imunossupressão permite a instalação de infecções oportunistas, e a cavidade bucal é um local ideal para a presença dessas lesões ${ }^{1,4,9}$, cuja prevalência em pacientes pediátricos é, em média, de $63 \%^{7,11}$.

O curso da infecção pelo HIV em crianças tem distinções daquele observado em pacientes adultos, devido, principalmente, ao momento precoce em que ocorre a contaminação pelo vírus e à imaturidade não apenas do sistema imunológico como também dos demais sistemas do corpo ${ }^{2,7}$. O espectro das lesões bucais em crianças infectadas pelo HIV diferencia-se daquele dos adultos, sendo necessária mais atenção aos pacientes pediátricos para registrar todas as lesões bucais, em especial, pela resposta inesperada aos tratamentos convencionais $\mathrm{s}^{1,4}$.

As lesões bucais mais comumente encontradas nesses pacientes foram cárie ${ }^{1-4,7,12}$; candidíase $^{1,3-7}$; leucoplasia pilosa ${ }^{1,3,5,7,11}$; infecção pelo vírus herpes simples ${ }^{1,3-5,7,9-11}$; doenças periodontais ${ }^{1,4,7,11}$; hipertrofia da glândula parótida ${ }^{1,3,4,7}$; sarcoma de Kapo$\mathrm{si}^{1,5-7,9-11}$; xerostomia ${ }^{4,7}$; ulceração aftosa recorrente $^{1,4,7}$ e infecções bacterianas na mucosa bucal ${ }^{1,4,6,7}$.

Tem sido alvo de discussão e objeto de muitos estudos a ocorrência de cárie dentária em crianças infectadas pelo $\mathrm{HIV}^{1-3,4,7,12}$. Em um estudo de $2009^{2}$, foram avaliados 133 pacientes de um ambulatório de Aids pediátrica do Rio de Janeiro e 85 pacientes da clínica de Odontopediatria da mesma instituição, por meio do índice de cárie (ceo/CPOD), não havendo diferenças estatisticamente significativas nesse índice, se comparados os dois grupos (ceo: 6,4 e 8,0; CPOD: 1,0 e 1,4 para o primeiro e o segundo grupo, respectivamente). Ambos apresentaram exposição a fatores de risco e alta prevalência de cárie. $\mathrm{O}$ hábito de ingestão de sacarose entre as refeições, avaliado pela entrevista aos responsáveis em crianças sem evidência clínica de imunossupressão, mostrou-se importante, associado à alta prevalência de cárie na dentição decídua.

Consensualmente, a candidíase é uma das manifestações bucais mais comuns em pacientes infectados pelo $\mathrm{HIV}^{1,3-7}$. Trata-se de infecção fúngica causada pelo Candida albicans. Contudo, nesses pacientes, parece estar também associada à presen- ça de C. dubliniensis ${ }^{1}$. São encontrados vários valores de prevalência para a manifestação, variando de 5,4 a $90 \%{ }^{1,3,4,6,7}$.

As variantes da candidíase mais comuns apresentaram-se como queilite angular ou na sua forma pseudomembranosa ${ }^{4,5}$. Sua prevalência está relacionada a valores mais baixos de linfócitos TCD4+, sendo, então, um importante indicador de comprometimento imunológico ${ }^{1}$.

$\mathrm{O}$ acometimento das glândulas salivares associadas ao HIV é um fenômeno bastante comum nas crianças, podendo ocorrer em qualquer estágio da infecção. A hipertrofia da glândula parótida tem sido relatada em diversos estudos na literatura ${ }^{1,3,4,7}$. Esse aumento da glândula associada ao HIV ainda não foi completamente elucidado, entretanto pode estar relacionado às mudanças no estágio imunológico do paciente e, consequentemente, com a diminuição do número de células $\mathrm{CD} 4+{ }^{1}$. A prevalência de acometimento varia entre 2 e $60 \%$ dos pacientes ${ }^{1,3,7}$.

O sarcoma de Kaposi, apesar de ser raramente encontrado em pacientes pediátricos, é a neoplasia passível de acometer crianças infectadas pelo $\mathrm{HIV}^{1,5-}$ 7,9-11. Evidências têm demonstrado forte associação do sarcoma de Kaposi com o herpes vírus humano tipo 8 (HHV8), que parece ter papel central na fisiopatologia desse neoplasma ${ }^{10}$. O sarcoma de Kaposi associado a crianças infectadas pelo HIV tem sido descrito, mais frequentemente, em países do continente africano ${ }^{7,9,11}$. Foram encontradas prevalências de $18,6^{6}$ a $22 \%$ para esse sarcoma em pacientes infectados pelo HIV. Outro estudo de $2011^{6}$ obteve valor de $0,2 \%$, possivelmente em virtude de um grande número de indivíduos avaliados em sua coorte e da nacionalidade das crianças, todas brasileiras.

A leucoplasia pilosa é descrita, também, como importante precursora da infecção pelo HIV, sendo relevante, portanto, no diagnóstico da doença ou como marcador da ineficácia do tratamento ${ }^{1,3,7}$. Sua etiologia está relacionada à presença do Epstein-barr vírus - $(\mathrm{EBV})^{1,5,11} \mathrm{e}$ do HHV8 ${ }^{5}$. A prevalência varia de 0 a $22,5 \%$ nas crianças infectadas ${ }^{1,11}$.

A infecção pelo vírus herpes simples é relatada em pacientes pediátricos infectados pelo HIV $\mathrm{HI}^{1,3-7,9-11}$. Sendo uma infecção viral causada, principalmente, pelo HSV-1 (Human Simplex Virus 1; vírus do herpes simples tipo 1), provoca lesões bucais e manifestações sistêmicas encontradas com frequência em crianças soropositivas e possui tendência de recidiva ${ }^{1}$. A forma HHV8, como descrito anteriormente, é fortemente associada às lesões de sarcoma de Kaposi ${ }^{5,11}$. Na literatura, é relatada a prevalência do HHV8 de 2,1\% $\%^{6}$ até $3 \%$. Em um estudo de $2013^{11}$, o HHV-7 e o HHV-6 foram encontrados em $68 \%$ e $18 \%$, respectivamente, dos swabs orais utilizados para o exame de crianças infectadas pelo HIV.

Várias formas da doença periodontal são observadas nesses pacientes ${ }^{1,4,71}$, em especial as formas 
agudas e ulceronecrosantes, com uma prevalência de $5 \%$ para periodontites nos pacientes pediátricos ${ }^{4,7}$.

Uma das formas da doença periodontal classicamente observada em pacientes pediátricos infectados pelo HIV é o eritema gengival linear, que se trata de uma manifestação exclusiva desses pacientes $^{1,3,4}$. Não apresenta fatores locais associados e não responde bem ao tratamento convencional (raspagem radicular e profilaxia), tendo sido de $27 \%$ a prevalência encontrada ${ }^{1,3}$.

Como consequência da terapia antirretroviral altamente ativa (HAART), da hiperplasia da glândula parótida ou de ambas, a xerostomia é comumente vista nessas crianças ${ }^{4,7}$. Está fortemente associada à progressão da infecção pelo HIV, contribuindo para a ocorrência de cárie e da doença periodontal ${ }^{4,7}$.

As ulcerações aftosas recorrentes ainda aparecem como manifestações bucais comuns da infecção pediátrica pelo $\mathrm{HIV}^{1,4,7}$. Sua prevalência é de $15 \%{ }^{4}$ e, em indivíduos imunologicamente comprometidos, elas se apresentam como úlceras de maior diâmetro e mais duradouras ${ }^{7}$.

Entre as infecções bacterianas na mucosa bucal, há as gengivites e periodontites, anteriormente descritas, além de várias infecções oportunistas, como a tuberculose, com manifestações bucais decorrentes da baixa resposta imunológica a esses agentes ${ }^{1,3-7}$.

Em um estudo em que se avaliou 90 pacientes, a experiência de cárie foi também determinada pelo índice ceod/CPOD ${ }^{7}$. Informações sobre alimentação e hábitos de higiene bucal foram obtidas por meio de um questionário; a média do ceod foi de 2,6 $( \pm 3,6)^{7}$.

$\mathrm{O}$ uso da HAART esteve associado com a prevalência de cáries rampantes, e os pacientes pediátricos infectados pelo HIV mostraram maior risco de cáries na dentadura decídua, existindo controvérsias no que tange à susceptibilidade à cárie dentária nesses pacientes ${ }^{3}$. A sua maior prevalência parece estar mais associada à utilização de medicamentos antirretrovirais, pela sua própria composição ou administração, combinada a alimentos contendo sacarose $^{1,3,4,7,12}$. Não obstante, a imunossupressão predispõe os indivíduos às infecções bacterianas, estando a cárie dentro desse espectro ${ }^{1,3-6}$.

Nesse contexto, é fundamental a detecção das manifestações bucais em crianças infectadas pelo HIV, a fim de se chegar ao correto diagnóstico e maximizar o prognóstico pela iniciação do tratamento antirretroviral ${ }^{7,12}$. Aliado a isso, torna-se imprescindível a conscientização dos responsáveis por essas crianças da importância da saúde bucal para uma maior e melhor sobrevida, bem como fazê-los entender que são participantes ativos para a melhoria da saúde bucal e qualidade de vida desses pacientes $^{3,7,12}$.
Vários estudos ressaltam, também, a importância da participação do odontopediatra em equipes multiprofissionais que atendem crianças e adolescentes infectados pelo HIV, a fim de se promover adequação nas condições de saúde bucal e, consequentemente, uma melhoria na qualidade de vida desses pacientes ${ }^{1,3,4,11,12}$.

\section{Conclusões}

Dada a relevância do assunto e tendo em vista a pequena quantidade de estudos em literatura recente que reportem sobre as condições bucais de crianças infectadas pelo HIV, são necessárias a conscientização e a centralização de esforços, a fim de aumentar o número dessas pesquisas para amparar e aliciar programas voltados para a exploração das lesões bucais no diagnóstico e prognóstico da infecção pelo HIV.

As lesões bucais em crianças infectadas pelo HIV diferenciam-se daquelas dos adultos, sendo necessária mais atenção do cirurgião-dentista a esses pacientes, no intuito de registrá-las no momento do diagnóstico. Dentre essas manifestações, as mais comumente encontradas, segundo a literatura consultada, foram: cárie dentária, candidose, queilite angular, eritema linear gengival, patologias nas glândulas salivares, sarcoma de Kaposi, linfoma não Hodgkin, doenças fúngicas, doenças virais, doença periodontal, hipoplasia de esmalte e condiloma acuminado.

Os estudos demonstraram a importância da participação do odontopediatra em equipes multiprofissionais que atendem crianças infectadas pelo $\mathrm{HIV}$, com vistas a garantir-lhes uma melhor qualidade de vida.

\section{Abstract}

Introduction: when the Acquired Immunodeficiency Syndrome (Aids) affects individuals aged from zero to 13 years, it is named pediatric Aids, and it may show some significant manifestations in the oral cavity. $\mathrm{Ob}$ jective: this study aimed to investigate, through a systematic literature review, the main oral manifestations in pediatric patients infected with HIV. Methods: searching DOAJ (Directory of Open Access Journals), SciELO, and VHL (Virtual Health Library - Bireme) databases, eleven articles published between 2008 and 2013 were selected. Results: in the assessment performed by the Jadad scale, six out of nine analytical studies had a similar or higher score than three, and were considered of good methodological expression. The main oral manifestations present in pediatric patients infected with HIV found in the selected articles were: dental caries, candidiasis, angular cheilitis, linear gingival erythema, mucosal lesions, salivary gland diseases, Kaposi's sarcoma, non-Hodgkin lymphoma, fungal diseases, viral 
diseases, periodontal disease, enamel hypoplasia, and condyloma acuminata. Conclusion: the importance of dentists in multidisciplinary teams for the care of these children was evidenced by this study, and of the performance of further studies to report on oral conditions in HIV infected children.

Keywords: HIV. Pediatric dentistry. Pediatrics. Oral manifestations.

\section{Endereço para correspondência:}

Stéphanie Quadros Tonelli Rua Pedro Montes Claros, 56 - Centro

39400-059 Montes Claros - MG

Fone: (38) 91502443

E-mail: stephanie tonelli@hotmail.com

Recebido: 04/11/2013. Aceito: 03/12/2013.

\section{Referências}

1. Alves FBT, Czlusniak GD, Dal'maso AMS, Shimizu KH, Verri MA. Lesões estomatológicas em crianças HIV positivas e suas implicações clínicas. Arq Odontol 2009; 45:191-8.

2. Kelly A, Soares LF, Pomarico L, Souza IPR. Risco e atividade de cárie em crianças com e sem infecção pelo HIV. Rev Gaucha Odontol 2009; 57:217-22.

3. Dávila ME, Gil M. Manifestaciones orales y caries dental em niños expuestos al virus de inmunodeficiencia humana. Rev Salud Pública 2011; 13:833-43.

4. Carrasco C, William GG, Elena M, Vilma T. Comparacion de la presencia de queilitis angular en niños $\mathrm{VIH}(+)$ y $\mathrm{VIH}(-)$, hijos de madres seropositivas. Acta Odontol 2008; 46:1-9.

5. Oladokun R, Kolude B, Ogun G, Brown B, Osinusi K. Kaposi sarcoma in HIV positive nigerian children: a case series. World Journal of AIDS 2011; 1:63-9.

6. Ramos JR, Matida LH, Hearst N, Heukelbach J. Opportunistic illnesses in Brazilian children with AIDS: results from two national cohort studies, 1983-2007. AIDS Res Therapy 2011; 8:1-7.

7. Sales-Peres SHC, Mapengo MAA, Moura-Grec PG, Marsicano JA, Sales-Peres AC, Sales-Peres A. Oral manifestations in HIV+ children in Mozambique. Ciênc Saúde Coletiva 2012; 17:55-60.

8. Jadad AR, Moore RA, Carrol D. Assessing the quality of reports of randomized clinical trials: is blinding necessary? Control Clin Trials 1996; 17:1-12.

9. Zanoni BC, Phungula T, Zanoni HM, France H, Feeney ME. Risk factors associated with increased mortality among HIV infected children initiating antiretroviral therapy (ART) in South Africa. Plos One 2011; 6:1-6.

10. Burningham Z, Hashibe M, Spector L, Schiffman JD. The epidemiology of sarcoma. Clin Sarc Res 2012; 2:1-16.

11. Pinheiro RS, Ferreira DC, Nóbrega F, Santos NSO, Souza IPR, Castro GFBA. Current status of herpesvirus identification in the oral cavity of HIV-infected children. Rev Soc Bras Med Trop 2013; 46:15-9.

12. Machado FC, Souza IPR, Tura LFR, Castro GF. Adesão a um programa de atenção à saúde bucal para crianças e adolescentes infectados pelo HIV e atitude dos responsáveis. Ciênc Saúde Coletiva 2008; 13:1851-7. 